\title{
Evaluations of the serological test in the diagnosis of 2019 novel coronavirus (SARS-CoV-2) infections during the COVID-19 outbreak
}

\author{
Dachuan Lin ${ }^{1}$ - Lei Liu ${ }^{2}$ Mingxia Zhang ${ }^{2}$ - Yunlong Hu ${ }^{1}$. Qianting Yang ${ }^{2}$. Jiubiao Guo ${ }^{1}$ - Youchao Dai ${ }^{1}$. \\ Yuzhong Xu ${ }^{3} \cdot \mathrm{Yi} \mathrm{Cai}^{1} \cdot$ Xinchun Chen $^{1} \cdot$ Kaisong Huang ${ }^{1}$ (D) $\cdot$ Zheng Zhang $^{2}$
}

Received: 22 April 2020 / Accepted: 2 July 2020 / Published online: 17 July 2020

(C) Springer-Verlag GmbH Germany, part of Springer Nature 2020

\begin{abstract}
We developed a chemiluminescence immunoassay method based on the recombinant nucleocapsid antigen and assessed its performance for the clinical diagnosis of severe acute respiratory syndrome coronavirus (SARS-CoV)-2 infections by detecting SARS-CoV-2-specific IgM and IgG antibodies in patients. Full-length recombinant nucleocapsid antigen and tosyl magnetic beads were used to develop the chemiluminescence immunoassay approach. Plasmas from 29 healthy cohorts, 51 tuberculosis patients, and 79 confirmed SARS-CoV-2 patients were employed to evaluate the chemiluminescence immunoassay method performance for the clinical diagnosis of SARS-CoV-2 infections. A commercial ELISA kit (Darui Biotech, China) using the same nucleocapsid antigen was used for the in-parallel comparison with our chemiluminescence immunoassay method. The IgM and IgG manner of testing in the chemiluminescence immunoassay method showed a sensitivity and specificity of $60.76 \%(95 \%$ CI 49.1 to 71.6 ) and $92.25 \%$ (95\% CI 83.4 to 97.2 ) and $82.28 \%$ (95\% CI 72.1 to 90.0 ) and $97.5 \%$ (95\% CI 91.3 to 99.7), respectively. Higher sensitivity and specificity were observed in the chemiluminescence immunoassay method compared with the Darui Biotech ELISA kit. The developed high sensitivity and specificity chemiluminescence immunoassay IgG testing method combined with the RT-PCR approach can improve the clinical diagnosis for SARS-CoV-2 infections and thus contribute to the control of COVID-19 expansion.
\end{abstract}

Keywords SARS-CoV-2 $\cdot$ Serological testing $\cdot$ Chemiluminescence immunoassay $\cdot \operatorname{IgM}$ and IgG

Dachuan Lin and Lei Liu contributed equally to this work.

Electronic supplementary material The online version of this article (https://doi.org/10.1007/s10096-020-03978-6) contains supplementary material, which is available to authorized users.

Kaisong Huang

kaisong@szu.edu.cn

Zheng Zhang

zhangzheng1975@aliyun.com

1 Guangdong Provincial Key Laboratory of Regional Immunity and Disease, Department of Pathogen Biology, School of Medicine, Shenzhen University, Shenzhen, China

2 National Clinical Research Center for Infectious Diseases, Guangdong Key Lab for Diagnosis \&Treatment of Emerging Infectious Diseases, Shenzhen Third People's Hospital, Southern University of Science and Technology, Shenzhen, China

3 Department of Clinical Laboratory, Shenzhen Baoan People's Hospital, The Second Affiliated Hospital of Shenzhen University, Shenzhen, China

\section{Introduction}

Coronavirus is a group of enveloped, non-segmented positive-sense RNA virus belonging to the family of Coronaviridae and order of Nidovirales and capable of infecting both humans and animals including cattle, swine, chicken, cat, horse, camels, rodent, bats, and snakes [1-3]. Based on genetic properties, coronavirus was further divided into four genera, namely, Alphacoronavirus, Betacoronavirus, Gammacoronavirus, and Deltacoronavirus [4]. Prior to December 2019, a total of six coronaviruses were documented to cause disease in humans, including two strains from Alphacoronavirus (HCoV-229E and HKU-NL63) and four from Betacoronavirus subfamily (HCoV-OC43, HCoVHKU1, SARS-CoV, and MERS-CoV) [5-10]. The severe acute respiratory syndrome coronavirus (SARS-CoV) and Middle East respiratory syndrome coronavirus (MERS-CoV) are among the most well-described, as they led directly to two deadly large-scale outbreaks globally, with 8096 infection 
cases and roughly $10 \%$ mortality and 2494 cases and $34.4 \%$ mortality, respectively $[9,10]$.

Recently, the outbreak of a severe pneumonia COVID-19 was confirmed to be caused by the 2019 novel coronavirus infections (SARS-CoV-2), which was originated from a seafood wholesale market in Wuhan, China [11]. So far, this novel coronavirus has spread throughout the whole of China and over 198 countries globally, causing more than two million laboratory-confirmed cases of infections and 165,107 deaths, posing a great threat to the global public health (http://2019ncov.chinacdc.cn/2019-nCov/). Besides, there are still numerous suspected cases and a myriad of medical monitoring people who were quarantined in specialized hospitals or at homes because of their previous epidemiological link to confirmed SARS-CoV-2 patients. All of these circumstances put an extreme burden on the emergency, hospital, and public health system particularly in the epidemic zone worldwide. Therefore, a timely, sensitive, and accurate diagnosis approach is urgently needed and of pivotal importance for surveillances of disease dissemination and the prevention of further expansions. Conventional diagnosis methods such as virus culture and microscopic analysis are generally time-consuming and labor-intensive with limited sensitivity $[12,13]$. In contrast, the last decade-emerged molecular biologic and serologic approaches, such as TaqMan real-time reverse transcription polymerase chain reaction (RT-PCR), enzyme-linked immunosorbent assay, colloidal gold immunochromatography, and direct chemiluminescence immunoassay (CLIA), can be developed into a rapid and effective tool for detections of respiratory pathogens infections, even though in certain circumstances, molecular biologic method like RT-PCR had a low sensitivity for specimens from the upper respiratory tract [14-17].

In this study, we developed a chemiluminescence immunoassay method to specifically detect the SARS-CoV-2-induced IgM and IgG antibody using the recombinant nucleocapsid (YP_009724397.2) and evaluated its sensitivity and specificity in detecting COVID-19 patients. High sensitivity and specificity results indicate that this chemiluminescence immunoassay method in combination with RT-PCR method can serve as a highly sensitive and accurate tool for the screen and diagnosis of SARS-CoV-2 infections in the community.

\section{Material and methods}

\section{Participants and specimens}

In Shenzhen city, China, patients infected by SARS-CoV-2 were all eventually admitted into a specialized hospital (the Third People's Hospital of Shenzhen) for quarantines and treatments. In this study, a total of 29 healthy individuals, 51 tuberculosis patients, and 79 SARS-CoV-2 patients were enrolled for serological testing. Seventy-nine COVID-19 patients were randomly enrolled from the abovementioned specialized hospital, and their SARS-CoV-2 infections were confirmed according to the official guidelines for diagnosis and treatment of 2019 novel coronavirus infections issued by the National Health Commission of the People's Republic of China. More specifically, the SARS-CoV-2 infections were confirmed by combinations of epidemiological risk, clinical features, and positive detections of SARS-CoV-2 RNA in respiratory specimens using the National Medical Production Administration authorized GeneoDX kit. COVID-19 patients' blood samples were immediately collected when admitted into the Third People's Hospital of Shenzhen. Basic characteristics, including days after onset and ages for those patients, were summarized in Supplementary Table S2. Fifty-one tuberculosis patients were enrolled from the Shenzhen Bao'an hospital, and their mycobacterium tuberculosis infections were previously confirmed by sputum smear acid-fast bacilli analysis, chest radiograph, and the QuantiFERON®-TB Gold test. Twenty-nine healthy people were recruited from the Shenzhen University in which the campus has been strictly closed during the COVID-19 epidemic period. All healthy cohorts and tuberculosis patients control had no epidemiological risks like traveling to the epidemic area or contact with confirmed COVID-19 patients. They were also persistently negative for SARS-CoV-2 RNA detections in at least three respiratory specimens' tests using the abovementioned GeneoDX kit. None of the healthy cohorts and tuberculosis patients control was found to be SARSCoV-2 positive in 2-month follow-up period after blood collection. Peripheral blood samples for all the above participants were collected into sodium heparin-containing tubes, and then the plasma was separated by centrifugations $(800 \mathrm{~g} \times$ $10 \mathrm{~min}$ ) for immediate testing or stored at $-80{ }^{\circ} \mathrm{C}$ until used. Verbal informed consent was obtained from all individual participants.

\section{SARS-CoV-2 nucleic acid}

Total nucleic acid for collected respiratory specimens was extracted in BSL-3 laboratory using the nucleic acid extraction and purification kit from Huayin Bio-Tech (Shenzhen, China) according to the manufacturer protocol. The detections of SARS-CoV-2 RNA were performed using the National Medical Production Administration-authorized GeneoDX kit (TaqMan RT-PCR method, targeting the ORF1ab and N genes) according to the manufacturer's instructions.

\section{Development of chemiluminescence immunoassay and test procedures}

After the transcription of extracted SARS-CoV-2 genome RNA into the cDNA, the coding regions (Accession 
Number: YP 009724397.2) were then amplified and cloned into the pET30a vector. The recombinant full-length nucleocapsid antigen was expressed in engineering Escherichia coli BL21 (DE3) strains and purified using the Ni-NTA resin (Darui Biotech, China) (Fig. S1). The purified nucleocapsid was also further verified by His-tag monoclonal antibody (data not shown). Magnetic beads Magnosphere ${ }^{\mathrm{TM}}$ MS300 used in this study are commercially available in the JSR Corporation, Tokyo, Japan. Recombinant nucleocapsid antigens were coupled to these tosyl magnetic beads using the catalytic reagent solution (3-M ammonium sulfate/0.1-M borate buffer, $\mathrm{pH}$ 9.5) according to the manufacture's instruction, and the resultant beads were further blocked by $0.05 \%$ BSA for $6 \mathrm{~h}$ at $37{ }^{\circ} \mathrm{C}$. The following testing and detection procedure were automated on a chemical immunoluminescence analyzer ACCRE6 (Tianshen Tech, Shenzhen, China). Fifty microliters of pure plasma was firstly incubated with the magnetic beads that were coupled with antigens for 5 min at $37{ }^{\circ} \mathrm{C}$. Subsequently, the unbound substance was gently removed and then washed by Tris buffer for three times. Alkaline phosphatase-labeled antihuman immunoglobulin $(50 \mu \mathrm{g} / \mathrm{ml})$ was added and further incubated for $5 \mathrm{~min}$ at $37{ }^{\circ} \mathrm{C}$ in the MES buffer. After three times of washing to remove unbound materials, the Lumigen APS- 5 substrate $(50 \mu \mathrm{g} / \mathrm{ml})$ was added. Ultimately, the light signal was measured by the photomultiplier in ACCRE6 (Tianshen Tech, Shenzhen, China) as relative light units, and the whole testing can be completed in $23 \mathrm{~min}$. Confirmed SARS-CoV-2 positive plasma and negative plasma from initial trial testings were used as controls in each later set test.

\section{Detections of IgG and IgM by a commercial ELISA kit}

In parallel testing, the commercial enzyme-linked immunosorbent assay kit (Darui Biotech, CHINA) for detections of the anti-SARS-CoV-2 IgG and IgM antibody was used to measure the SARS-CoV-2 antibody level in the abovementioned COVID-19 patients and control individuals. For the principle of this ELISA kit, the specific SARS-CoV-2 nucleocapsid protein and antihuman IgM monoclonal antibody were firstly coated on the plates, respectively. Subsequently, the $100 \mu \mathrm{l}$ of 100 -fold diluted plasma was added and then incubated for $60 \mathrm{~min}$ at $37{ }^{\circ} \mathrm{C}$. After five washes with PBST buffer, the horseradish peroxidase (HRP)-labeled mouse antihuman IgG antibody or HRPlabeled SARS-CoV-2 nucleocapsid antigen was added for 30-min incubation at $37^{\circ} \mathrm{C}$. A $50-\mu \mathrm{l}$ TMB substrate was then added for 15-min incubation after washing by PBST buffer. The stopping solution was eventually added to suspend the reaction, and OD 450/630 values were immediately measured using the Varioskan LUX ${ }^{\mathrm{TM}}$ multimode microplate reader. The cutoff values for positive were set based on the manufacturer's recommendations.

\section{Statistical analysis}

All statistical analysis was performed in GraphPad Prism 7 software. The one-way ANOVA test was used to analyze the average RLU values difference between different participant groups. The chip-square and Fisher's exact test was used to compare the difference between the analyzed groups. The difference was considered significant when a $p$ value is $<0.05$.

\section{Results}

\section{Detections of IgG and IgM antibodies induced by SARS-CoV-2 in plasma and overall specificity and sensitivity assessments}

To assess the specificity and sensitivity of the chemiluminescence immunoassay method developed based on the recombinant nucleocapsid antigen, plasmas from 29 healthy individuals, 51 tuberculosis patients, and 79 confirmed SARS-CoV-2 patients were employed for testing. More than six-fold and eight-fold higher average RLU (relative light units) values were observed in the SARS-CoV-2 patients' group for the IgM testing in comparison with that of the healthy cohort and tuberculosis patients (Fig. 1a). This average RLU difference is more dramatic when it comes to the $\operatorname{IgG}$ testing reaching 60- and 70-fold increase in SARS-CoV-2 patients in comparison with the healthy and tuberculosis group, respectively (Fig. 1b). A receiver operating characteristic curve was then obtained based on these RLUs for the SARS-CoV-2 patients and control group that consists of a healthy cohort and tuberculosis patients. According to the ROC curve and analysis results, we recommend a cutoff setting for IgM (RLU 244802) and IgG (RLU 336697), in which the calculated sensitivity and specificity for IgM were $60.76 \%$ (95\% CI 49.1 to 71.6) and $92.25 \%$ (95\% CI 83.4 to 97.2 ) and $82.28 \%$ (95\% CI 72.1 to 90.0 ) and $97.5 \%$ (95\% CI 91.3 to 99.7 ) for IgG.

Based on this cutoff and using the IgM testing, we identified 6 cases and 48 cases as SARS-CoV-2 positive from the control group (80 cases) and the SARS-CoV-2 confirmed group (79 cases), respectively (Table 1 ). In contrast, we only detected two false-positive cases from the control group, and 65 cases were identified as SARS-CoV-2 positive from 79 COVID-19 confirmed patients when using the IgG testing. When combining IgM and IgG detections for SARS-CoV-2 infections, the sensitivity and specificity can reach $84.81 \%$ and $91.25 \%$, respectively (Table 1 ).

\section{The links between disease onset time, ages, and IgM and IgG productions and detection efficiency}

To explore whether the onset time was significantly linked with the detection sensitivity by this serological 

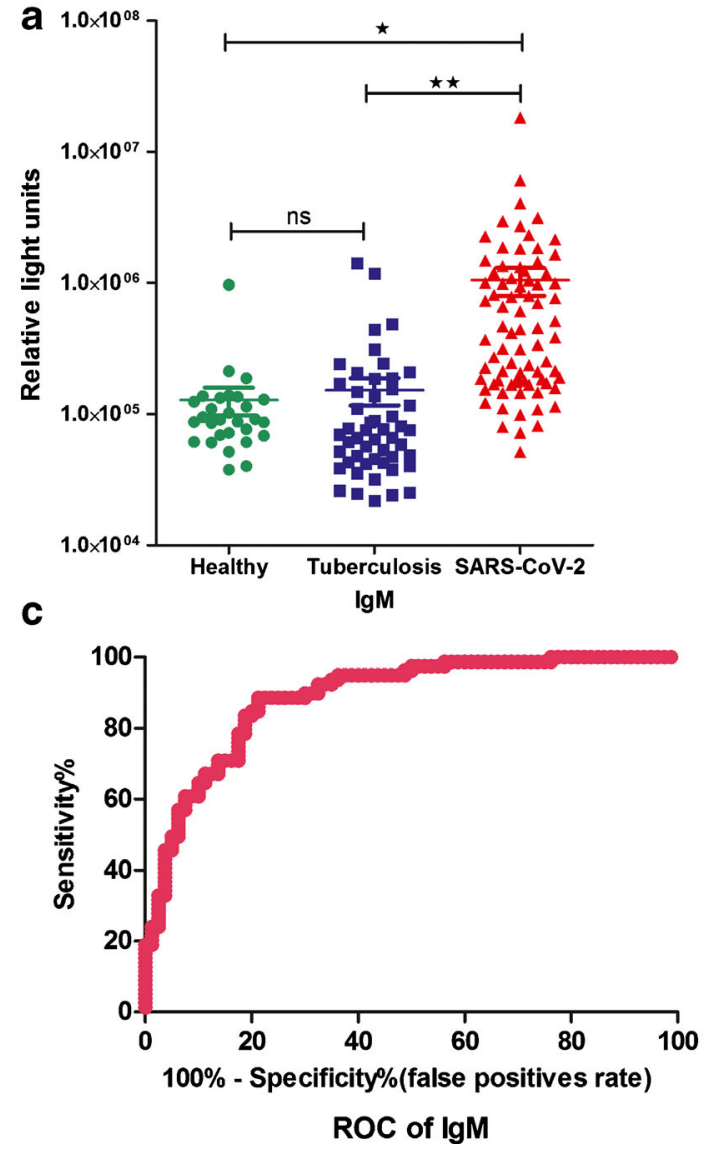

Fig. 1 Detections and measurements of the SARS-CoV-2 IgM and IgG antibody in healthy people, tuberculosis patients, and SARS-CoV-2 confirmed patients using the chemiluminescence immunoassay method (a and $\mathbf{b})$. The average results were expressed as mean \pm SEM of all

chemiluminescence method, the comparison and statistical analysis of the sensitivity rates between different onset time patient categories was undertaken. No statistically significant difference was observed between the $\operatorname{IgM}$ and $\operatorname{IgG}$ testing results in the patients with the onset time less than 1 week,

Table 1 Evaluations of a chemiluminescence immunoassay method for diagnosis of SARS-CoV-2 by detections of specific IgM and IgG in the patient's serum

\begin{tabular}{lllll}
\hline $\begin{array}{l}\text { Participants category } \\
\text { /total cases }\end{array}$ & $\begin{array}{l}\text { IgM- } \\
\text { positive } \\
\text { cases/(\%) }\end{array}$ & $\begin{array}{l}\text { IgG- } \\
\text { positive } \\
\text { cases/(\%) }\end{array}$ & $\begin{array}{l}\text { IgM- or } \\
\text { IgG- } \\
\text { positive } \\
\text { cases/(\%) }\end{array}$ & $\begin{array}{l}\text { IgM- and } \\
\text { IgG- } \\
\text { positive } \\
\text { cases (\%) }\end{array}$ \\
\hline $\begin{array}{l}\text { False-positive in } \\
\text { healthy cohorts } \\
\text { and tuberculosis } \\
\text { patients/total 80 } \\
\text { cases }\end{array}$ & $6 / 7.5 \%$ & $2 / 2.50 \%$ & $7 / 8.75 \%$ & $1 / 1.25 \%$ \\
$\begin{array}{l}\text { SARS-CoV-2 } \\
\text { confirmed patients }\end{array}$ & $48 / 60.76 \%$ & $65 / 82.28 \%$ & $67 / 84.81 \%$ & $46 / 58.22 \%$ \\
\begin{tabular}{l} 
/total 79 cases \\
\hline
\end{tabular} & & & & \\
\hline
\end{tabular}
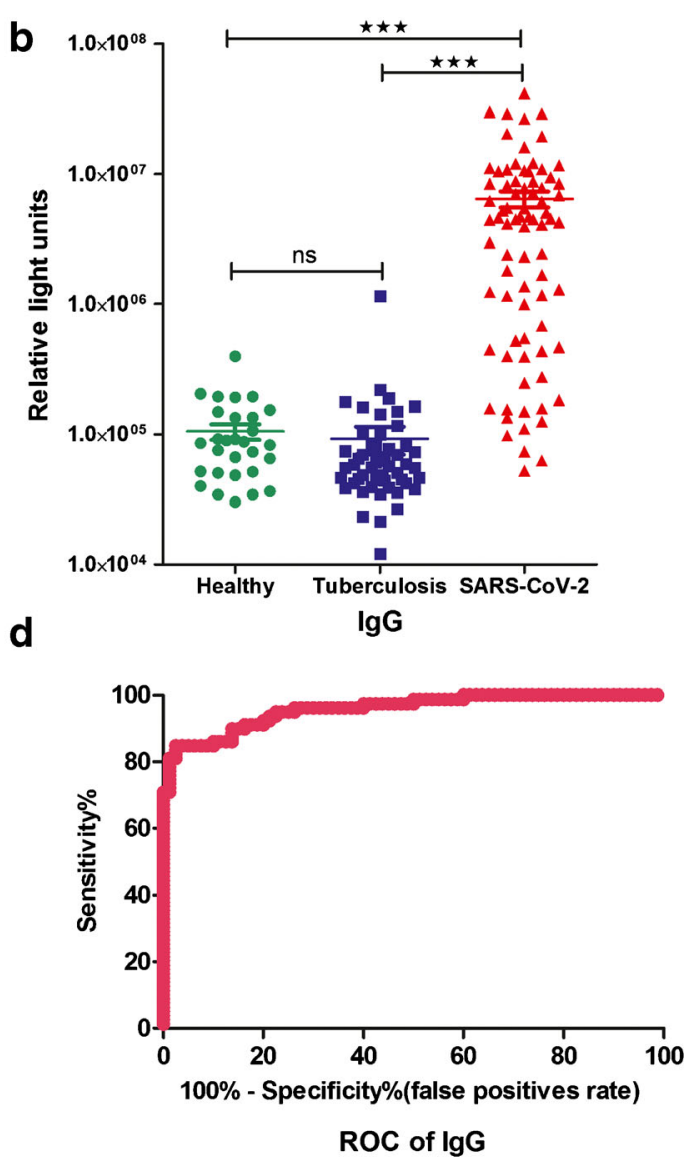

individuals. Receiver operating characteristic curves for IgM (c) and IgG (d) were obtained based on the RLU for the SARS-CoV-2 patient group and the control group consisting of healthy cohorts and tuberculosis patients

although two more cases from 12 cases were detected by $\operatorname{IgG}$ testing manner compared with that of IgM testing method (Table 2, $p$ value $>0.05$ ). However, the IgG testing manner exhibited a significant high detection rate in patients with the onset time between 8 and 14 days compared with that of IgM testing manner $(p$ value $<0.05$ ). A higher detection rate by the IgG testing manner was also seen in patients with the onset of symptoms over 2 weeks compared with that of the counterpart IgM testing ( $p$ value $=0.054$ ). In addition, we also examined the detection rates between the different age groups, and significantly lower detection rates in both $\operatorname{IgM}$ and $\operatorname{IgG}$ testing manner for individual group younger than 18 years old were observed compared with that of people aging from 18 to 65 ( $p$ value $<0.05$ ) (Supplementary Table $\mathrm{S} 1$ ). No statistically significant differences were observed for male and female groups.

\section{Comparisons with other ELISA kits}

To further characterize the patient's immune response to the SARS-CoV-2 antigens and to prove the feasibility of the 
Table 2 Comparison of SARS$\mathrm{CoV}-2$ detection results in patients with different onset time between the IgM and IgG approach

\begin{tabular}{llllll}
\hline $\begin{array}{l}\text { Days after } \\
\text { onset }\end{array}$ & $\begin{array}{l}\text { Total } \\
\text { cases }\end{array}$ & $\begin{array}{l}\text { IgM-positive } \\
\text { cases/(\%) }\end{array}$ & $\begin{array}{l}\text { IgG-positive } \\
\text { cases/(\%) }\end{array}$ & $\begin{array}{l}\text { IgM- or IgG-positive } \\
\text { cases/(\%) }\end{array}$ & $\begin{array}{l}\text { IgM-and IgG-positive } \\
\text { cases }(\%)\end{array}$ \\
\hline $0-3$ & 4 & $3 / 75 \%$ & $4 / 100 \%$ & $4 / 100 \%$ & $3 / 75 \%$ \\
$4-7$ & 8 & $3 / 37.5 \%$ & $4 / 50.0 \%$ & $4 / 50.0 \%$ & $3 / 37.5 \%$ \\
$8-14$ & 33 & $15 / 45.45 \%$ & $24 / 72.73 \%$ & $26 / 78.79 \%$ & $13 / 16.45 \%$ \\
$>14$ & 34 & $27 / 79.41 \%$ & $33 / 97.06 \%$ & $33 / 97.06 \%$ & $27 / 79.41 \%$ \\
\hline
\end{tabular}

practical application of this serological testing kit in clinical diagnosis, 64 paired plasmas from the abovementioned control cohorts and 65 COVID-19 patients were also examined using a recently developed commercial available ELISA Kit. Fourteen false-positive cases $(21 \%)$ were identified by IgM testing in the ELISA method, while only six false-positive cases were detected by our chemiluminescence immunoassay IgM testing in these 64 paired control group people. In contrast, a much lower false-positive rate was seen in IgG testing in both methods. Compared with the ELISA kit, a higher detection rate for SARS-CoV-2 in both IgM and IgG testing manners was seen in our chemiluminescence method, suggesting a higher sensitivity of our approach compared with the tested ELISA kit (Table $3, \operatorname{IgG} p$ value $<0.05, \operatorname{IgM} p$ value $=0.11$ )

\section{Discussion}

Compared with the conventional virological methods, the molecular biologic TaqMan RT-PCR method is currently widely used for the clinical diagnosis of respiratory pathogen infections particularly in the initial phase of the disease because of its high specificity property [18, 19]. However, a relatively limited sensitivity (less than 50\%) in single upper respiratory specimen testing has been commonly reported in SARS-CoV2 detections [20]. Furthermore, consecutive failed detections of SARS-CoV-2 RNA from upper respiratory specimens in patients with apparent clinical symptoms were also sporadically reported, in which the positive results can only be achieved by collecting the bronchoalveolar lavage fluid for re-testing [21]. Hence, a sensitive serological diagnosis method may serve as a handy compensation tool for current clinical diagnosis situations. Our results demonstrated that the IgG testing is helpful for the clinical diagnosis of SARS-CoV2 , as a relatively higher specificity and sensitivity were observed in our chemiluminescence method particularly in patients with onset over 2 weeks. In the humoral immune response, the antibody $\operatorname{IgM}$ was generally produced earlier than the IgG isotype as the IgM can be expressed without the isotype switching. Unexpectedly, no statistically significant detection rate difference was observed between these two antibodies isotype testing manner in patients with the onset of disease less than 7 days. Since we only had 12 cases in this category, the limited samples may serve as a confounding factor for this phenomenon. In contrast, a significantly higher detection rate was seen for IgG testing manner in patients with symptom onset over 1 week compared with that of the IgM testing manner (57/67 vs 42/67). Since the IgM and IgG testing way used the same pure recombinant antigen and coupling condition (Supplementary Fig. S1), the detection sensitivity difference was more likely linked to intrinsic traits and concentration differences of the specific antibody isotype in patients.

We noted that four patients with clinical symptoms of less than 4 days were simultaneously positive in both $\operatorname{IgM}$ and $\operatorname{IgG}$ testings. A close examination of their medical record reveals that all of them had previous contact with confirmed SARSCoV-2 individuals in at least 16 days ago, pointing to the possibility that they were asymptomatically infected by the virus for a certain time already. When comparing the detection rates in different age groups by our method, a significantly lower detection rate in both IgM and IgG testing manner for the individual group younger than 18 years old was observed
Table 3 Detection differences between the chemiluminescence and ELISA method

\begin{tabular}{|c|c|c|c|c|}
\hline \multirow[t]{2}{*}{ Methods } & \multicolumn{2}{|c|}{ Control group (total 64 cases) } & \multicolumn{2}{|c|}{$\begin{array}{l}\text { SARS-CoV-2 confirmed patients (total } 65 \\
\text { cases) }\end{array}$} \\
\hline & $\begin{array}{l}\text { IgM false-positive } \\
1 \%\end{array}$ & $\begin{array}{l}\text { IgG false-positive } \\
1 \%\end{array}$ & $\begin{array}{l}\text { IgM-positive } \\
1 \%\end{array}$ & $\begin{array}{l}\text { IgG-positive } \\
1 \%\end{array}$ \\
\hline ELISA & $14 / 21.8 \%$ & $0 / 0 \%$ & $30 / 46.1 \%$ & $15 / 23 \%$ \\
\hline Chemiluminescence & $6 / 9.38 \%$ & $2 / 3.1 \%$ & $40 / 61.54 \%$ & $53 / 81.5 \%$ \\
\hline Identified in both & $1 / 1.56 \%$ & $0 / 0 \%$ & $25 / 38.46 \%$ & $15 / 23 \%$ \\
\hline
\end{tabular}


compared with that of people aging from 18 to 65 . An indepth look of their symptom onset time indicates that all of them were less than 14 days, with six people even less 7 days when collected samples (Supplementary Table S2). Hence, the lower detection rate for these twelve people younger than 18 years old was likely associated with less or no production of antibodies in them at the time when we collected the peripheral bloods.

In our parallel performance testing, the same antigen of nucleocapsid protein was used in both the commercially available ELISA kit and our chemiluminescence immunoassay. Unexpectedly, a significantly higher sensitivity was observed in our method compared with the ELISA kit. This sensitivity difference may be partially attributed to the difference in the plasma amount for the first incubation step. On the other side, the intrinsic method difference including the aspect of binding surface interaction and mode of separations of the unbound material can also contribute to the sensitivity difference in the chemiluminescence immunoassay and ELISA method.

In conclusion, in this study, we developed and evaluated a serological chemiluminescence immunoassay testing technique for the clinical diagnosis of SARS-CoV-2 infections using the recombinant nucleocapsid antigen. This high sensitivity and specificity chemiluminescence immunoassay $\operatorname{IgG}$ testing method combined with the RT-PCR approach can doubtlessly improve the clinical diagnosis for SARS-CoV-2 significantly and contribute to the control of COVID-19 expansion globally.

Acknowledgment We are very grateful to Prof. Yingsong $\mathrm{Wu}$ at Southern Medical University for his kind gifts of several proteins for our trail test and helpful suggestions for the whole project.

Funding Information This work is supported by the Guangdong Provincial Science and Technology Program (No. 2019b030301009), the National Natural Science Funds of China (81802060), the National Science and Technology Major Project (2017ZX10201301), and Guangdong province Universities for Special Research Program for COVID-19 control (2020kzdx1175).

\section{Compliance with ethical standards}

Conflict of interest The authors declare that they have no conflict of interest.

Ethical approval The internal use of collected samples for diagnoses of etiological agents and serological research was approved by the Ethical Committee in the Third People's Hospital of Shenzhen (SZTHEC2016001). Verbal informed consent was obtained from all individual participants.

\section{References}

1. Wang LF, Shi Z, Zhang S, Field H, Daszak P, Eaton BT (2006) Review of bats and SARS. Emerg Infect Dis 12:1834-1840
2. Lim XF, Lee CB, Pascoe SM, How CB, Chan S, Tan JH, Yang X, Zhou P, Shi Z, Sessions OM, Wang LF, Ng LC, Anderson DE, Yap G (2019) Detection and characterization of a novel bat-borne coronavirus in Singapore using multiple molecular approaches. J Gen Virol 100:1363-1374

3. Fehr AR, Perlman S (2015) Coronaviruses: an overview of their replication and pathogenesis. Methods Mol Biol 1282:1-23

4. Chan JF, To KK, Tse H, Jin DY, Yuen KY (2013) Interspecies transmission and emergence of novel viruses: lessons from bats and birds. Trends Microbiol 21:544-555

5. St-Jean JR, Jacomy H, Desforges M, Vabret A, Freymuth F, Talbot PJ (2004) Human respiratory coronavirus OC43: genetic stability and neuroinvasion. J Virol 78:8824-8834

6. Chiu SS, Chan KH, Chu KW, Kwan SW, Guan Y, Poon LL, Peiris JS (2005) Human coronavirus NL63 infection and other coronavirus infections in children hospitalized with acute respiratory disease in Hong Kong, China. Clin Infect Dis 40:1721-1729

7. Woo PC, Lau SK, Yip CC, Huang Y, Yuen KY (2009) More and More Coronaviruses: Human Coronavirus HKU1. Viruses 1:57-71

8. Vassilara F, Spyridaki A, Pothitos G, Deliveliotou A, Papadopoulos A (2018) A rare case of human coronavirus 229e associated with acute respiratory distress syndrome in a healthy adult. Case Rep Infect Dis 2018:6796839

9. Chan JF, Lau SK, To KK, Cheng VC, Woo PC, Yuen KY (2015) Middle East respiratory syndrome coronavirus: another zoonotic betacoronavirus causing SARS-like disease. Clin Microbiol Rev 28:465-522

10. Cheng VC, Lau SK, Woo PC, Yuen KY (2007) Severe acute respiratory syndrome coronavirus as an agent of emerging and reemerging infection. Clin Microbiol Rev 20:660-694

11. Zhu N, Zhang D, Wang W, Li X, Yang B, Song J, Zhao X, Huang B, Shi W, Lu R, Niu P, Zhan F, Ma X, Wang D, Xu W, Wu G, Gao GF, Tan W, China Novel Coronavirus I, Research T (2020) A novel coronavirus from patients with pneumonia in China, 2019. N Engl J Med. https://doi.org/10.1056/NEJMoa2001017

12. Sarasini A, Percivalle E, Rovida F, Campanini G, Genini E, Torsellini M, Paolucci S, Baldanti F, Marchi A, Grazia Revello M, Gerna G (2006) Detection and pathogenicity of human metapneumovirus respiratory infection in pediatric Italian patients during a winter-spring season. J Clin Virol 35:59-68

13. Rose RM, Pinkston P, O'Donnell C, Jensen WA (1987) Viral infection of the lower respiratory tract. Clin Chest Med 8:405-418

14. Vemula SV, Zhao J, Liu J, Wang X, Biswas S, Hewlett I (2016) Current approaches for diagnosis of influenza virus infections in humans. Viruses 8:96

15. Goy G, Croxatto A, Posfay-Barbe KM, Gervaix A, Greub G (2009) Development of a real-time PCR for the specific detection of Waddlia chondrophila in clinical samples. Eur J Clin Microbiol Infect Dis 28:1483-1486

16. Mahony JB, Petrich A, Smieja M (2011) Molecular diagnosis of respiratory virus infections. Crit Rev Clin Lab Sci 48:217-249

17. Poon LL, Chan KH, Wong OK, Yam WC, Yuen KY, Guan Y, Lo YM, Peiris JS (2003) Early diagnosis of SARS coronavirus infection by real time RT-PCR. J Clin Virol 28:233-238

18. Li J, Li W, Mao L, Hao F, Yang L, Zhang W, Jiang J (2016) Rapid detection of novel caprine parainfluenza virus type 3 (CPIV3) using a TaqMan-based RT-qPCR. J Virol Methods 236:126-131

19. Weinberg GA, Schnabel KC, Erdman DD, Prill MM, Iwane MK, Shelley LM, Whitaker BL, Szilagyi PG, Hall CB (2013) Field evaluation of TaqMan Array Card (TAC) for the simultaneous detection of multiple respiratory viruses in children with acute respiratory infection. J Clin Virol 57:254-260

20. Xie C, Jiang L, Huang G, Pu H, Gong B, Lin H, Ma S, Chen X, Long B, Si G, Yu H, Jiang L, Yang X, Shi Y, Yang Z (2020) Comparison of different samples for 2019 novel coronavirus detection by nucleic acid amplification tests. Int J Infect Dis 93:264-267 
21. Tan FR, Qiu YL, Xu Z (2020) Bronchoalveolar lavage fluid was used to diagnose two cases of 2019-nCoV infection. Zhonghua Jie He He Hu Xi Za Zhi 43:337-339
Publisher's note Springer Nature remains neutral with regard to jurisdictional claims in published maps and institutional affiliations. 